\title{
The Efficacy of Radiotherapy in the Treatment of Hepatocellular Carcinoma with Distant Organ Metastasis
}

\author{
Lei Chen (i), 1,2 Zhiwen Wang, ${ }^{3}$ Songlin Song, ${ }^{1,2}$ Tao Sun,,2 Yanqiao Ren, ${ }^{1,2}$ \\ Weihua Zhang, ${ }^{1,2}$ Mingfu Wang, ${ }^{4}$ Yiming Liu $\mathbb{D}^{1,2}$ and Chuansheng Zheng $\mathbb{D}^{1,2}$ \\ ${ }^{1}$ Department of Radiology, Union Hospital, Tongji Medical College, Huazhong University of Science and Technology, \\ Wuhan 430022, China \\ ${ }^{2}$ Department of Interventional Radiology, Union Hospital, Tongji Medical College, \\ Huazhong University of Science and Technology, Wuhan 430022, China \\ ${ }^{3}$ Department of Cardiology, Union Hospital, Tongji Medical College, Huazhong University of Science and Technology, \\ Wuhan 430022, China \\ ${ }^{4}$ Department of Radiology, The Third People's Hospital of Hubei Province, Wuhan 430033, China
}

Correspondence should be addressed to Yiming Liu; 304271574@qq.com and Chuansheng Zheng; hqzcsxh@sina.com

Received 4 August 2021; Revised 25 October 2021; Accepted 1 November 2021; Published 17 November 2021

Academic Editor: Ozkan Kanat

Copyright (c) 2021 Lei Chen et al. This is an open access article distributed under the Creative Commons Attribution License, which permits unrestricted use, distribution, and reproduction in any medium, provided the original work is properly cited.

Background. Recently, radiotherapy has been used in the treatment of hepatocellular carcinoma (HCC). However, there is no study analyzing the efficacy of radiotherapy in cases of advanced HCC. The objective of this investigation was to determine the efficacy of radiotherapy in patients with HCC invading distant organs. Methods. The data of 2342 patients diagnosed between 2010 and 2015 with HCC invading distant organs were extracted from the SEER database. Propensity score matching (PSM) was used to reduce selection bias. Results. Before PSM, the median overall survival (mOS) and median cancer-specific survival (mCSS) in the radiotherapy group ( $\mathrm{mOS}=5$ months, $95 \% \mathrm{CI}: 4.5-5.5$; $\mathrm{mCSS}=5$ months, $95 \% \mathrm{CI}: 4.4-5.6$ ) were longer than those in the nonradiotherapy group (mOS $=3$ months, $95 \%$ CI: $2.8-3.2$; mCSS $=3$ months, $95 \%$ CI: $2.8-3.2$; both $P<0.001$ ). After PSM, mOS in the radiotherapy group ( 5 months, $95 \%$ CI: 4.5-5.5) was longer than that in the nonradiotherapy group (3 months, $95 \%$ CI: 2.6-3.4; $P<0.001$ ), and the mCSS in the radiotherapy group (5 months, 95\% CI: 4.4-5.6) was longer than that in the nonradiotherapy group (3 months, 95\% CI: 2.6-3.4; $P<0.001$ ). Before PSM, the multivariate analysis showed that all-cause and cancer-specific mortality rates were higher in the nonradiotherapy group than in the radiotherapy group. The adjusted Cox regression analysis for subgroups showed that, in the nonradiotherapy group, patients with bone metastases and multiorgan metastases had a worse survival than those in the radiotherapy group. Conclusion. HCC patients with metastases to distant organs obtain survival benefit from radiotherapy, particularly patients with bone metastases and multiorgan metastases.

\section{Introduction}

Hepatocellular carcinoma (HCC) is one of the most common cancers with one of the highest fatality rates [1]. Patients with early HCC can get survival benefits from transplantation, surgery, or ablation [2-5]. Transarterial chemoembolization (TACE) can prolong the overall survival of patients with intermediate HCC, and some studies have shown the survival benefits of TACE in patients with advanced HCC $[6,7]$. However, due to the lack of high-level evidence, TACE is currently not considered the first-line treatment for advanced HCC [8]. Sorafenib and lenvatinib are recommended for this category of patients, but these drugs are expensive, and the response rate is low [8-10]. Although in the last year a progress in early diagnosis of HCC has been reached, the changed scenario characterized by emerging etiologies such as metabolic causes of cirrhosis has led to a high rate of patients who receive HCC diagnosis in advance stages characterized by extrahepatic spread, as recently reported [11]. This changed scenario needs to enhance treatment strategies for advanced HCC, including radiotherapy. 
Radiotherapy includes external and external radiotherapy, and both forms are being used in the treatment of several types of solid tumors [12-15]. Lin J. and coworkers reported that HCC patients with portal vein tumor thrombus (PVTT) could obtain survival benefits from iodine- 125 seeds scaffold [16]. Another meta-analysis yielded similar results [17]. Some studies showed that external radiotherapy prolonged the overall survival (OS) of patients with different types of tumors that invaded distant organs [18-20]. A random controlled trial documented that external radiotherapy could prolong the median OS of patients with oligometastasis from different primary tumors [21]. However, for several decades, radiotherapy has not been recommended for the treatment of HCC because the radiation could damage healthy liver tissue. However, with the advancement of technology, the accuracy of external radiotherapy is more precise, allowing the clinicians to avoid damage to the normal liver tissue around the tumor. Several recent studies demonstrated that patients with HCC could gain survival benefits from radiotherapy [22-24].

The survival prognosis of patients with HCC that invades distant organs is dismal. Although radiotherapy benefits patients with different primary tumors invading distant organs, there are no studies addressing the question of whether patients with HCC metastasizing to distant organs can obtain survival benefits from radiotherapy. Thus, we compared the efficacy of radiotherapy with other treatments in HCC patients with the tumor invading distant organs.

\section{Materials and Methods}

2.1. Patient selection. Data used in the study originated from the Surveillance, Epidemiology, and End Results (SEER) database and were extracted using the SEER $*$ Stat software. The SEER database collects data on cancer cases from various locations and sources throughout the United States and includes approximately $28 \%$ of the United States population. The present analysis utilized the SEER data on patients diagnosed with HCC. The study was approved by the institutional Ethics Committee. The written informed consent was waived, since anonymized data were obtained from the national database.

The inclusion criteria were as follows: (1) patients diagnosed as HCC (International Classification of Disease for Oncology, Third Edition (ICD-O-3), histology codes 8170/ 3-8175/3) between 2010 and 2015; (2) patients aged between 30 and 84 years; (3) patients having extrahepatic metastases (including multiorgan invasion); (4) patients for whom the information on radiotherapy treatment (yes or no) was available; and (5) patients having a known survival time (those with survival codes 0 and 999 were excluded) (Figure 1).

2.2. Definition of the Endpoints. The endpoints of the study were overall survival (OS) and cancer-specific survival (CSS). OS was defined as the interval from the time patients were diagnosed with HCC to the time of death caused by any reason. CSS was defined as the interval from the time of HCC diagnosis to the time of death caused by the cancer.
2.3. Statistical Analysis. The study included twelve baseline factors, and the continuous variables were converted to categorical variables. Chi-square test and Fisher's test were used to compare the difference of baseline factors between the radiotherapy and nonradiotherapy groups. The survival curves were plotted using the Kaplan-Meier method, and the survival was compared by log-rank test. Cox proportional risk model was used to exclude the potential factors which might influence the survival of patients in the two groups. For subgroups multivariate regression analysis, the adjusted Cox proportional risk model was used to reduce the effects of confounding factors on survival. The adjusted Cox regression analysis considered the age at diagnosis, gender, year of diagnosis, tumor grade, American Joint Committee on Cancer 7th edition (AJCC 7th) T stage, AJCC 7th $\mathrm{N}$ stage, tumor size, AFP, chemotherapy, number of tumors, race, marital status, and the type of surgery.

The factors of age at diagnosis, gender, AJCC 7th stage, metastatic organs, race, AFP, fibrosis scores, and chemotherapy were not balanced between the two groups. Thus, propensity score matching (PSM) including all factors analyzed in the study was used to balance the baseline factors. The optimal caliper of the PSM was set as 0.02, and 529 pairs of patients were generated by $1: 1$ ratio matching. After matching, all factors in the two groups were balanced. The statistical analysis was performed using the SPSS 24.0 (IBM, Chicago, IL, USA) and R 3.6.2 software. A $P$ value of less than 0.05 was considered statistically significant.

\section{Results}

3.1. Characteristics of Patients. A total of 2342 patients were included in the study. Among them, 647 patients received radiotherapy (radiotherapy group), and 1695 did not (nonradiotherapy group). In the radiotherapy group, 467 patients had bone metastases, 63 patients had lung metastases, 8 patients had brain metastases, and 109 patients had multiorgan metastases. In the nonradiotherapy group, 463 patients had bone metastases, 1035 patients had lung metastases, 17 patients had brain metastases, and 180 patients had multiorgan metastases. In the nonradiotherapy group, 746 patients received chemotherapy, 21 patients received ablation (10 patients received radiofrequency ablation and 11 patients received other ablations), and 30 patients received liver resection (Table 1).

3.2. Survival Analysis. Before PSM, the median OS (mOS) and median CSS (mCSS) in the radiotherapy group were 5 months (95\% CI: 4.5-5.5) and 5 months (95\% CI: 4.5-5.5), respectively. These values were longer than those in the nonradiotherapy group ( $\mathrm{mOS}=3$ months, 95\% CI: 2.8-3.2; mCSS $=3$ months, 95\% CI: 2.8-3.2; both $P<0.001$ ) (Figure 2). After PSM, the mOS (5 months, 95\% CI: 4.5-5.5) and mCSS (5 months, 95\% CI: 4.4-5.6) in the radiotherapy group were longer than the mOS (3 months, 95\% CI: 2.6-3.4; $P<0.001$ ) and mCSS (3 months, 95\% CI: 2.6-3.4; $P<0.001$ ) in the nonradiotherapy group (Figure 3 ). 


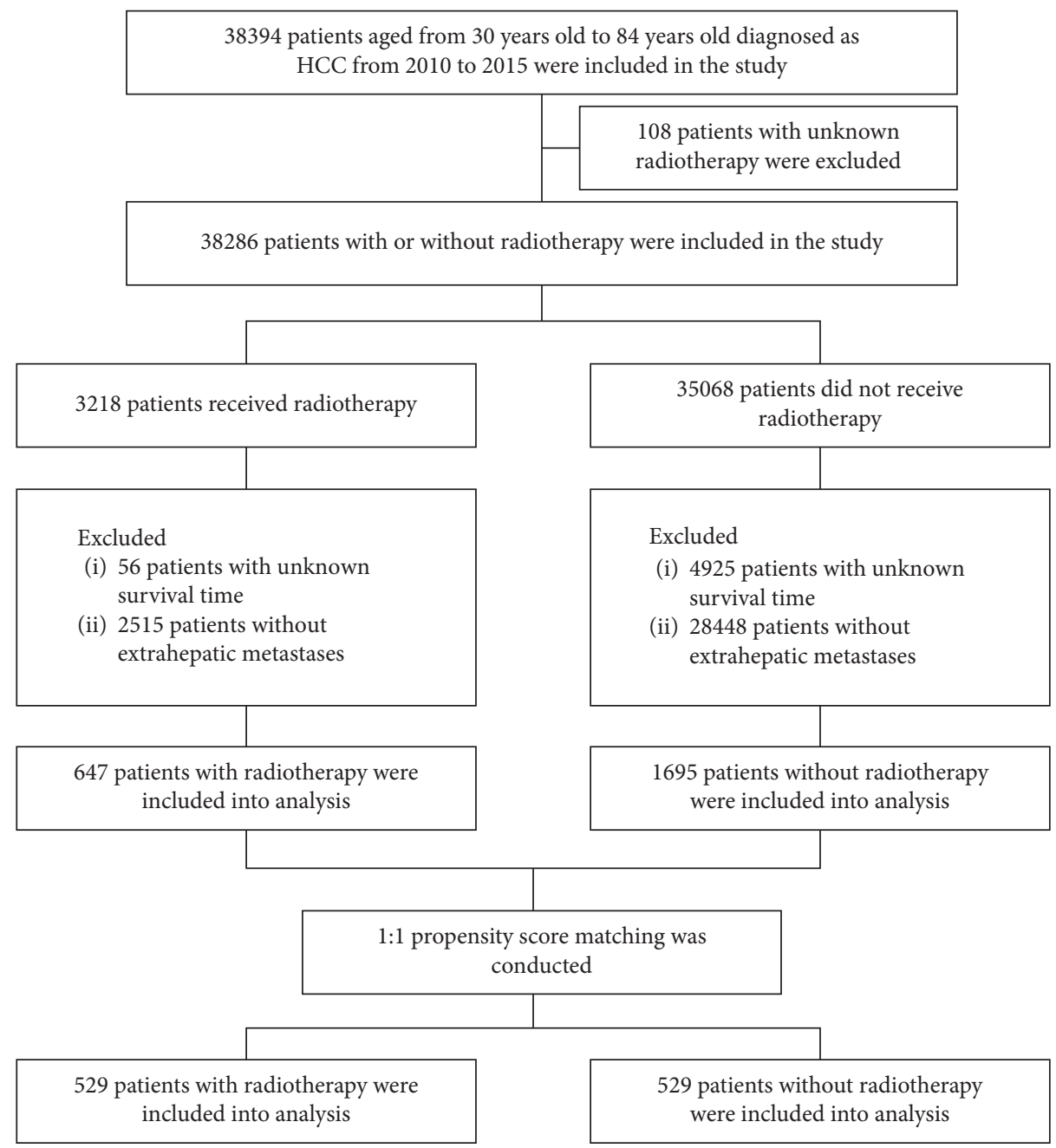

Figure 1: The flowchart of patient selection.

TABLE 1: Baseline characteristics of patients before matching and after matching.

\begin{tabular}{|c|c|c|c|c|c|c|}
\hline \multirow[b]{2}{*}{ Characteristics } & \multicolumn{3}{|c|}{ Before matching } & \multicolumn{3}{|c|}{ After matching } \\
\hline & $\begin{array}{l}\text { Radiotherapy } \\
\quad(N=647)\end{array}$ & $\begin{array}{l}\text { Nonradiotherapy } \\
\qquad(N=1695)\end{array}$ & $P$ value & $\begin{array}{l}\text { Radiotherapy } \\
\quad(N=529)\end{array}$ & $\begin{array}{l}\text { Nonradiotherapy } \\
\qquad(N=529)\end{array}$ & $\begin{array}{c}P \\
\text { value }\end{array}$ \\
\hline Age at diagnosis & & & 0.009 & & & 0.803 \\
\hline $30-44$ & 10 & 54 & & 10 & 13 & \\
\hline $45-59$ & 208 & 616 & & 178 & 180 & \\
\hline$\geq 60$ & 429 & 1025 & & 341 & 336 & \\
\hline Gender & & & 0.001 & & & 0.921 \\
\hline Male & 560 & 1369 & & 449 & 451 & \\
\hline Female & 87 & 326 & & 80 & 78 & \\
\hline Years of diagnosis & & & 0.063 & & & 0.389 \\
\hline 2010-2012 & 278 & 801 & & 243 & 257 & \\
\hline $2013-2015$ & 369 & 894 & & 286 & 272 & \\
\hline Tumor grade & & & 0.166 & & & 0.950 \\
\hline Well differentiated & 46 & 113 & & 39 & 35 & \\
\hline $\begin{array}{l}\text { Moderately } \\
\text { differentiated }\end{array}$ & 69 & 221 & & 60 & 55 & \\
\hline Poorly differentiated & 63 & 198 & & 52 & 49 & \\
\hline Undifferentiated & 3 & 16 & & 2 & 2 & \\
\hline Unknown & 466 & 1147 & & 376 & 388 & \\
\hline AJCC 7th T stage & & & 0.015 & & & 0.799 \\
\hline T0 & 7 & 9 & & 6 & 6 & \\
\hline
\end{tabular}


TABle 1: Continued.

\begin{tabular}{|c|c|c|c|c|c|c|}
\hline \multirow[b]{2}{*}{ Characteristics } & \multicolumn{3}{|c|}{ Before matching } & \multicolumn{3}{|c|}{ After matching } \\
\hline & $\begin{array}{l}\text { Radiotherapy } \\
\quad(N=647)\end{array}$ & $\begin{array}{c}\text { Nonradiotherapy } \\
\quad(N=1695)\end{array}$ & $P$ value & $\begin{array}{l}\text { Radiotherapy } \\
\quad(N=529)\end{array}$ & $\begin{array}{l}\text { Nonradiotherapy } \\
\quad(N=529)\end{array}$ & $\begin{array}{c}P \\
\text { value }\end{array}$ \\
\hline $\mathrm{T} 1$ & 165 & 334 & & 115 & 116 & \\
\hline $\mathrm{T} 2$ & 66 & 192 & & 59 & 52 & \\
\hline T3 & 228 & 621 & & 189 & 207 & \\
\hline $\mathrm{T} 4$ & 45 & 164 & & 36 & 39 & \\
\hline $\mathrm{TX}$ & 136 & 375 & & 124 & 109 & \\
\hline AJCC 7th $\mathrm{N}$ stage & & & 0.195 & & & 0.863 \\
\hline No & 434 & 1075 & & 339 & 343 & \\
\hline N1 & 106 & 327 & & 97 & 93 & \\
\hline NX & 107 & 293 & & 93 & 93 & \\
\hline Metastatic organs & & & $<0.001$ & & & 0.992 \\
\hline Bone & 467 & 463 & & 357 & 354 & \\
\hline Lung & 63 & 1035 & & 63 & 65 & \\
\hline Brain & 8 & 17 & & 8 & 9 & \\
\hline Multiple organs & 109 & 180 & & 101 & 101 & \\
\hline Tumor size & & & 0.052 & & & 0.689 \\
\hline No more than $5 \mathrm{~cm}$ & 153 & 325 & & 121 & 114 & \\
\hline Larger than $5 \mathrm{~cm}$ & 323 & 882 & & 258 & 272 & \\
\hline Unknown & 171 & 488 & & 150 & 143 & \\
\hline Tumor number & & & 0.186 & & & 0.777 \\
\hline 1 & 575 & 1472 & & 464 & 467 & \\
\hline$\geq 2$ & 72 & 223 & & 65 & 62 & \\
\hline Race & & & $<0.001$ & & & 0.106 \\
\hline White & 472 & 1074 & & 376 & 351 & \\
\hline Black & 104 & 317 & & 91 & 93 & \\
\hline Other/unknown & 71 & 304 & & 62 & 85 & \\
\hline Marital status & & & $<0.001$ & & & 0.952 \\
\hline Married & 352 & 767 & & 263 & 264 & \\
\hline Single & 273 & 825 & & 244 & 245 & \\
\hline Unknown & 22 & 103 & & 22 & 20 & \\
\hline AFP & & & 0.002 & & & 0.556 \\
\hline Positive & 404 & 1141 & & 338 & 350 & \\
\hline Negative & 75 & 182 & & 57 & 60 & \\
\hline Unknown & 168 & 372 & & 134 & 119 & \\
\hline Fibrosis scores & & & $<0.001$ & & & 0.447 \\
\hline $0-4$ & 34 & 62 & & 13 & 19 & \\
\hline $5-6$ & 89 & 242 & & 81 & 88 & \\
\hline Unknown & 524 & 1391 & & 435 & 422 & \\
\hline Chemotherapy & & & $<0.001$ & & & 0.324 \\
\hline Yes & 353 & 746 & & 257 & 241 & \\
\hline No/unknown & 294 & 949 & & 272 & 288 & \\
\hline Surgery & & & 0.435 & & & 0.881 \\
\hline Ablation & 12 & 21 & & 8 & 8 & \\
\hline Liver resection & 9 & 30 & & 7 & 9 & \\
\hline No & 626 & 1644 & & 514 & 512 & \\
\hline
\end{tabular}

3.3. Multivariate Regression Analysis. In the multivariate regression analysis before PSM, female patients, patients with poorly differentiated tumors, patients with the AJCC 7 th stage $\mathrm{T}$, and patients with larger tumor size had higher all-cause mortality rate and cancer-specific mortality rate. After excluding potential factors which might influence the survival, patients in the nonradiotherapy group had a higher all-cause mortality rate $(\mathrm{HR}=1.277,95 \% \mathrm{CI}$ : $1.146-1.424$; $P<0.001)$ and cancer-specific mortality rate $(\mathrm{HR}=1.315$, 95\% CI: $1.167-1.481 ; P<0.001)$ than patients in the radiotherapy group (Table 2 ).
3.4. Subgroup Analysis. Before PSM, the mOS (6 months, 95\% CI: 5.4-6.6) and mCSS (6 months, 95\% CI: 5.2-6.8) of patients with bone metastases in the radiotherapy group were longer than the mOS (3 months, 95\% CI: 2.5-3.5; $P<0.001$ ) and mCSS (3 months, 95\% CI: 2.7-3.3; $P<0.001$ ) in the nonradiotherapy group. The mOS (5 months, 95\% CI: 3.8-6.2) and mCSS (5 months, 95\% CI: 3.6-6.4) of patients with lung metastases in the radiotherapy group were longer than the mOS (2 months, 95\% CI: $1.8-2.2 ; P=0.011)$ and mCSS (2 months, 95\% CI: $1.8-2.2 ; P=0.043$ ) in the nonradiotherapy group. The mOS (4 months, 95\% CI: $3.3-4.7$ ) 

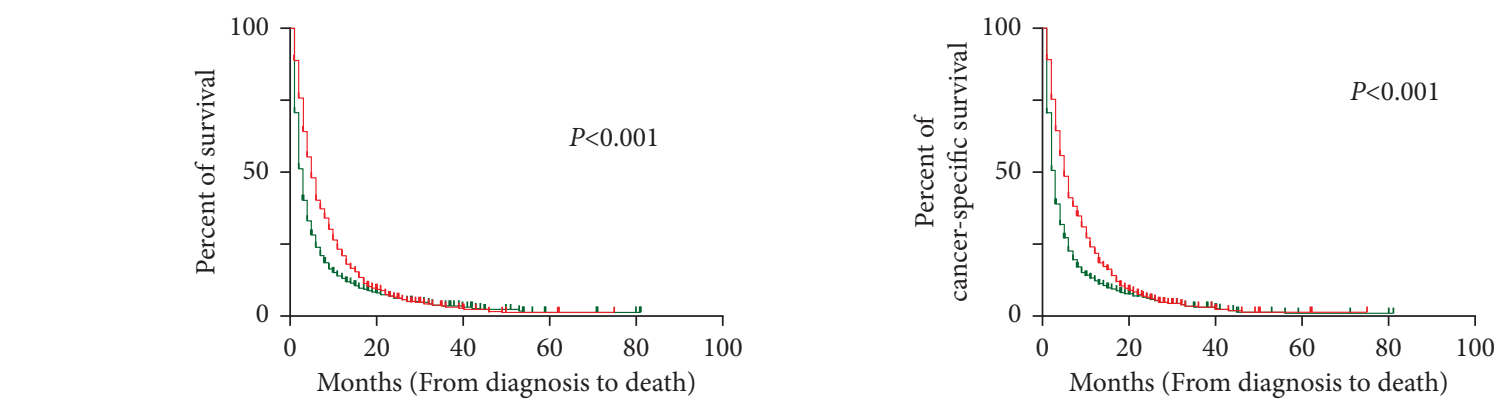

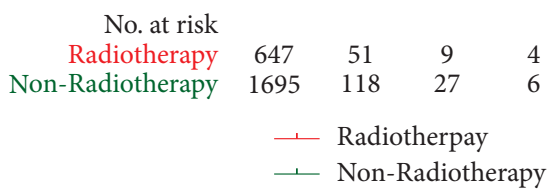

(a)
No. at risk Radiotherapy Non-Radiotherapy

$$
\begin{array}{cccccc}
560 & 43 & 8 & 4 & 0 & 0 \\
1380 & 90 & 17 & 4 & 2 & 0 \\
r & \\
r & \text { Radiotherpay } \\
\longrightarrow & \text { Non-Radiotherapy }
\end{array}
$$

(b)

FIGURE 2: Kaplan-Meier curves of OS and CSS in patients before PSM. (a) Kaplan-Meier curve of OS; (b) Kaplan-Meier curve of CSS.
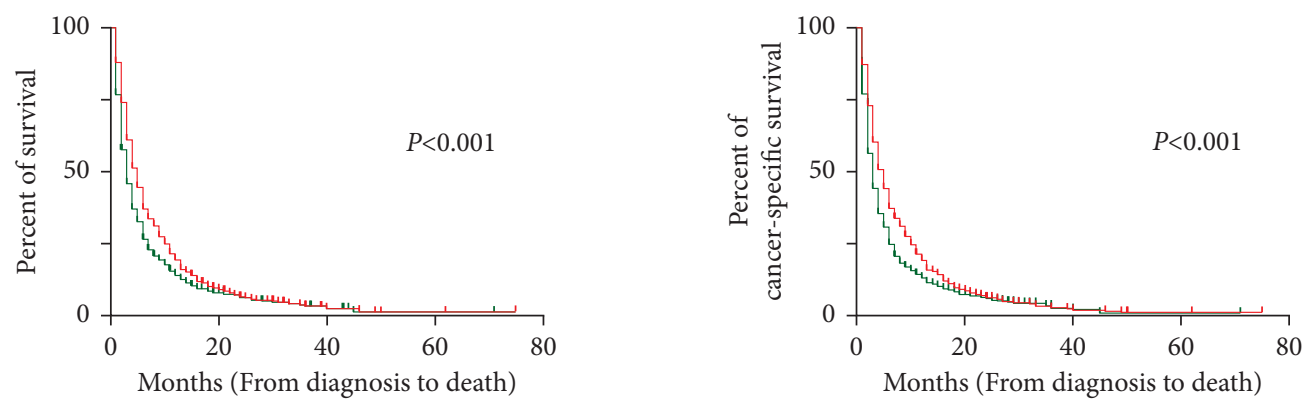

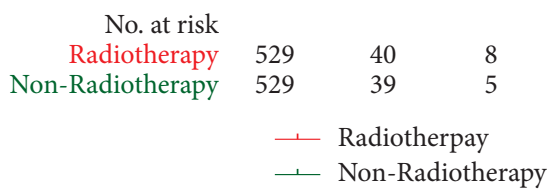

(a)
No. at risk Radiotherapy Non-Radiotherapy

$$
\begin{array}{ccc}
451 & 33 & 7 \\
445 & 31 & 4 \\
& & \text { Radiotherpay } \\
\longrightarrow & \text { Non-Radiothera }
\end{array}
$$

\begin{tabular}{|c|c|c|c|c|}
\hline \multirow{2}{*}{ Characteristics } & \multicolumn{2}{|c|}{ OS } & \multicolumn{2}{|c|}{ CSS } \\
\hline & HR (95\% CI) & $P$ value & HR (95\% CI) & $P$ value \\
\hline \multicolumn{5}{|l|}{ Age at diagnosis } \\
\hline $30-44$ & Reference & & Reference & \\
\hline $45-59$ & $1.369(1.043,1.797)$ & 0.024 & $1.244(0.934,1.657)$ & 0.136 \\
\hline$\geq 60$ & $1.388(1.060,1.818)$ & 0.017 & $1.290(0.971,1.714)$ & 0.079 \\
\hline \multicolumn{5}{|l|}{ Gender } \\
\hline Male & Reference & & Reference & \\
\hline Female & $0.870(0.776,0.974)$ & 0.016 & $0.893(0.788,1.013)$ & 0.079 \\
\hline \multicolumn{5}{|l|}{ Years of diagnosis } \\
\hline 2010-2012 & Reference & & Reference & \\
\hline 2013-2015 & $0.985(0.904,1.074)$ & 0.734 & $0.998(0.908,1.098)$ & 0.975 \\
\hline \multicolumn{5}{|l|}{ Tumor grade } \\
\hline Well differentiated & Reference & & Reference & \\
\hline Moderately differentiated & $1.066(0.869,1.306)$ & 0.542 & $0.989(0.783,1.249)$ & 0.928 \\
\hline
\end{tabular}

(b)

FIgURE 3: Kaplan-Meier curves of OS and CSS in patients after PSM. (a) Kaplan-Meier curve of OS; (b) Kaplan-Meier curve of CSS.

TABLe 2: Multivariable regression analysis for OS and CSS of all patients before PSM. 
TABle 2: Continued.

\begin{tabular}{|c|c|c|c|c|}
\hline \multirow{2}{*}{ Characteristics } & \multicolumn{2}{|c|}{ OS } & \multicolumn{2}{|c|}{ CSS } \\
\hline & HR (95\% CI) & $P$ value & HR (95\% CI) & $P$ value \\
\hline Poorly differentiated & $1.458(1.185,1.794)$ & $<0.001$ & $1.390(1.098,1.759)$ & 0.006 \\
\hline Undifferentiated & $1.231(0.757,2.001)$ & 0.401 & $1.379(0.799,2.380)$ & 0.249 \\
\hline Unknown & $1.179(0.992,1.400)$ & 0.062 & $1.129(0.928,1.374)$ & 0.226 \\
\hline \multicolumn{5}{|l|}{ AJCC 7th T stage } \\
\hline T0 & Reference & & Reference & \\
\hline $\mathrm{T} 1$ & $0.801(0.471,1.362)$ & 0.141 & $0.695(0.364,1.328)$ & 0.270 \\
\hline $\mathrm{T} 2$ & $1.053(0.619,1.792)$ & 0.850 & $0.918(0.481,1.753)$ & 0.796 \\
\hline $\mathrm{T} 3$ & $1.007(0.590,1.720)$ & 0.979 & $0.879(0.459,1.682)$ & 0.696 \\
\hline $\mathrm{T} 4$ & $1.020(0.589,1.766)$ & 0.973 & $0.901(0.465,1.747)$ & 0.758 \\
\hline TX & $0.858(0.498,1.477)$ & 0.580 & $0.732(0.378,1.417)$ & 0.355 \\
\hline \multicolumn{5}{|l|}{ AJCC 7th $\mathrm{N}$ stage } \\
\hline N0 & Reference & & Reference & \\
\hline N1 & $1.139(1.017,1.275)$ & 0.014 & $1.155(1.020,1.307)$ & 0.023 \\
\hline NX & $0.911(0.805,1.031)$ & 0.141 & $0.910(0.793,1.045)$ & 0.180 \\
\hline \multicolumn{5}{|l|}{ Metastatic organs } \\
\hline Bone & Reference & & Reference & \\
\hline Lung & $1.111(1.000,1.234)$ & 0.050 & $1.091(0.970,1.226)$ & 0.114 \\
\hline Brain & $0.986(0.660,1.473)$ & 0.945 & $0.887(0.545,1.442)$ & 0.628 \\
\hline Multiple organs & $1.314(1.145,1.506)$ & $<0.001$ & $1.301(1.123,1.508)$ & $<0.001$ \\
\hline \multicolumn{5}{|l|}{ Tumor size } \\
\hline No more than $5 \mathrm{~cm}$ & Reference & & Reference & \\
\hline Larger than $5 \mathrm{~cm}$ & $1.140(0.988,1.315)$ & 0.073 & $1.122(0.959,1.312)$ & 0.151 \\
\hline Unknown & $1.277(1.082,1.506)$ & 0.004 & $1.258(1.051,1.506)$ & 0.012 \\
\hline \multicolumn{5}{|l|}{ Tumor number } \\
\hline 1 & Reference & & Reference & \\
\hline$\geq 2$ & $0.892(0.782,1.017)$ & 0.088 & $0.591(0.382,0.917)$ & 0.019 \\
\hline \multicolumn{5}{|l|}{ Race } \\
\hline White & Reference & & Reference & \\
\hline Black & $0.993(0.887,1.112)$ & 0.916 & $0.976(0.861,1.106)$ & 0.703 \\
\hline Other/unknown & $0.995(0.882,1.124)$ & 0.942 & $1.050(0.919,1.199)$ & 0.417 \\
\hline \multicolumn{5}{|l|}{ Marital status } \\
\hline Married & Reference & & Reference & \\
\hline Single & $1.035(0.946,1.133)$ & 0.453 & $1.051(0.952,1.161)$ & 0.326 \\
\hline Unknown & $0.977(0.807,1.182)$ & 0.808 & $0.956(0.771,1.185)$ & 0.956 \\
\hline \multicolumn{5}{|l|}{ AFP } \\
\hline Positive & Reference & & Reference & \\
\hline Negative & $0.777(0.673,0.897)$ & 0.001 & $0.760(0.646,0.894)$ & 0.001 \\
\hline Unknown & $0.825(0.741,0.918)$ & $<0.001$ & $0.807(0.716,0.910)$ & $<0.001$ \\
\hline \multicolumn{5}{|l|}{ Fibrosis scores } \\
\hline $0-4$ & Reference & & Reference & \\
\hline $5-6$ & $1.002(0.784,1.279)$ & 0.990 & $1.001(0.762,1.314)$ & 0.995 \\
\hline Unknown & $1.203(0.964,1.500)$ & 0.101 & $1.268(0.991,1.623)$ & 0.059 \\
\hline \multicolumn{5}{|l|}{ Chemotherapy } \\
\hline Yes & Reference & & Reference & \\
\hline No/unknown & $1.602(1.466,1.750)$ & $<0.001$ & $1.617(1.466,1.783)$ & $<0.001$ \\
\hline \multicolumn{5}{|l|}{ Surgery } \\
\hline Ablation & Reference & & Reference & \\
\hline Liver resection & $0.995(0.589,1.682)$ & 0.985 & $1.040(0.596,1.817)$ & 0.889 \\
\hline No & $2.324(1.569,3.442)$ & $<0.001$ & $1.846(1.218,2.798)$ & 0.004 \\
\hline \multicolumn{5}{|l|}{ Treatment } \\
\hline Radiotherapy & Reference & & Reference & \\
\hline Nonradiotherapy & $1.277(1.146,1.424)$ & $<0.001$ & $1.315(1.167,1.481)$ & $<0.001$ \\
\hline
\end{tabular}

and mCSS (4 months, 95\% CI: 3.3-4.7) of patients with multiorgan metastases in the radiotherapy group were longer than the mOS ( 2 months, 95\% CI: $1.5-2.5 ; P=0.001$ ) and mCSS ( 2 months, 95\% CI: 1.5-2.5; $P<0.001)$ in the nonradiotherapy group. The mOS (5 months, 95\% CI: 3.4-6.6) and mCSS (6 months, 95\% CI: 3.4-8.6) of patients 
with fibrosis scores of $0-4$ in the radiotherapy group were not statistically significantly longer than the mOS (4 months, 95\% CI: $2.6-5.4 ; P=0.868$ ) and mCSS (4 months, 95\% CI: $3-5 ; P=0.527)$ in the nonradiotherapy group. The mOS (6 months, 95\% CI: 4.3-7.7) and mCSS (6 months, 95\% CI: 4.3-7.7) of patients with fibrosis scores of 5-6 in the radiotherapy group were longer than the mOS (3 months, $95 \%$ CI: 2.4-3.6; $P=0.066)$ and mCSS ( 3 months, 95\% CI: 2.3-3.7; $P=0.078$ ) in the nonradiotherapy group (Supplementary Figure 1).

After PSM, the mOS (5 months, 95\% CI: 4.4-5.6) and mCSS (6 months, 95\% CI: 5.2-6.8) of patients with bone metastases in the radiotherapy group were longer than the mOS (3 months, 95\% CI: $2.5-3.5 ; P=0.002)$ and mCSS (3 months, $95 \%$ CI: $2.4-3.6 ; P<0.001)$ in the nonradiotherapy group. The mOS (5 months, 95\% CI: 3.8-6.2) and mCSS (5 months, 95\% CI: 3.6-6.4) of patients with lung metastases in the radiotherapy group were longer than the mOS (3 months, 95\% CI: 1.9-4.1; $P=0.239$ ) and mCSS (3 months, 95\% CI: $2.2-3.8 ; \quad P=0.382$ ) of patients in the nonradiotherapy group, but these differences did not reach statistical significance. The mOS (3 months, 95\% CI: 2.3-3.7) and mCSS (3 months, 95\% CI: 2.3-3.7) of patients with multiorgan metastases in the radiotherapy group were longer than the mOS (2 months, 95\% CI: 1.4-2.4; $P=0.021$ ) and mCSS ( 3 months, 95\% CI: $2.4-3.6 ; P=0.025$ ) in the nonradiotherapy group. The mOS (2 months, 95\% CI: $0.5-3.5$ ) and mCSS (8 months, $95 \%$ CI: NA) of patients with fibrosis scores of $0-4$ in the radiotherapy group were not longer than the mOS (3 months, 95\% CI: $1.8-4.2 ; P=0.550$ ) and mCSS ( 3 months, 95\% CI: $1.7-4.3 ; P=0.596)$ in the nonradiotherapy group. The mOS (6 months, 95\% CI: 4.3-7.7) and mCSS (6 months, 95\% CI: 4-8) of patients with fibrosis scores of 5-6 in the radiotherapy group were not longer than the mOS (4 months, 95\% CI: $2.3-5.7 ; P=0.635$ ) and mCSS (4 months, 95\% CI: $2.2-5.8 ; P=0.346$ ) in the nonradiotherapy group, but these differences did not reach statistical significance (Supplementary Figure 2).

Before PSM, the adjusted Cox regression analysis showed that patients with bone metastases in the nonradiotherapy group had a higher all-cause mortality rate $(\mathrm{HR}=1.223,95 \% \mathrm{CI}: 1.062-1.410 ; P=0.005)$ and cancerspecific mortality rate $(\mathrm{HR}=1.326,95 \%$ CI: $1.138-1.545$; $P<0.001)$ than patients in the radiotherapy group. Patients with lung metastases in the nonradiotherapy group had a higher all-cause mortality rate $(\mathrm{HR}=1.394,95 \% \mathrm{CI}$ : 1.053-1.846; $P=0.02$ ) but not cancer-specific mortality rate $(\mathrm{HR}=1.305,95 \%$ CI: $0.961-1.773 ; P=0.088)$ than patients in the radiotherapy group. Patients with multiorgan metastases in the nonradiotherapy group had higher all-cause mortality rate $(\mathrm{HR}=1.387,95 \% \mathrm{CI}: 1.053-1.827 ; P=0.02)$ and cancer-specific mortality rate $(\mathrm{HR}=1.374,95 \% \mathrm{CI}$ : $1.018-1.855 ; P=0.038)$ than patients in the radiotherapy group. Radiotherapy did not reduce all-cause mortality rate and cancer-specific rate compared to no radiotherapy for patients with fibrosis scores of $0-4$ and fibrosis scores of 5-6 (all $P>0.05$ ) (Table 3).

After PSM, the adjusted Cox regression analysis showed that patients with bone metastases in the nonradiotherapy group had higher all-cause mortality rate $(\mathrm{HR}=1.198,95 \% \mathrm{CI}: 1.024-1.401 ; P=0.024)$ and cancerspecific mortality rate $(\mathrm{HR}=1.290,95 \% \mathrm{CI}: 1.089-1.528$; $P=0.003)$ than patients in the radiotherapy group. Patients with multiorgan metastases in the nonradiotherapy group had higher all-cause mortality rate $(\mathrm{HR}=1.438,95 \%$ CI: $1.040-1.989 ; P=0.028)$ and cancer-specific mortality rate $(\mathrm{HR}=1.459,95 \% \mathrm{CI}: 1.018-2.091 ; P=0.04)$ than patients in the radiotherapy group. Radiotherapy did not reduce all-cause mortality rate and cancer-specific rate compared to no radiotherapy for patients with fibrosis scores of $0-4$ and fibrosis scores of 5-6 (all $P>0.05$ ) (Supplementary Table 1).

\section{Discussion}

HCC invading distant organs is considered an advanced stage and predicts poor overall survival [8]. However, only a few investigations have focused on the treatments for patients with advanced HCC. Previous high-quality studies had shown that patients with multiple primary tumor oligometastases had better survival when treated with radiotherapy than when radiotherapy was not used $[21,25,26]$. Moreover, many clinical trials demonstrated that patients with HCC could also receive survival benefits from radiotherapy $[22,27]$. However, there was no study focusing on radiotherapy for HCC patients with extrahepatic metastases. Therefore, the present analysis was conducted to compare the survival of HCC patients with extrahepatic metastases who received radiotherapy with that of those that did not receive radiotherapy.

In the current study, HCC patients with extrahepatic metastases who were treated by radiotherapy had longer mOS and mCSS than patients who did not receive radiotherapy; this result was obtained before and after PSM. Previous research documented that the mOS of HCC patients with bone, adrenal gland, or peritoneum metastases who received sorafenib combined with internal radiotherapy was 13.9 months, which was longer than the mOS of 5 months found in the present work [28]. This difference may reflect the use of sorafenib as the first-line treatment of sorafenib, which could prolong the survival time of patients with advanced HCC. Another study on the efficacy of radiotherapy, conducted by Kim and coworkers, included 530 HCC patients with spine, pelvis, rib, or bone metastases. The results demonstrated that the mOS was 5.1 months, which was similar to the mOS found in the current study. In the study of Kim and coworkers, $63 \%$ of patients received chemotherapy or sorafenib treatment, a fraction higher than that in the current study (54.5\%). However, the patients in Kim et al.'s study did not receive other treatments (ablation or surgery), and, in the current study, $3 \%$ of patients were subjected to ablation or surgery, which might explain why mOS values were similar in both studies [29]. In the current study, the mOS and mCSS of patients treated with and without radiotherapy were compared, and the differences in $\mathrm{mOS}$ and $\mathrm{mCSS}$ between the two groups were similar before PSM and after PSM. This finding implied that patients' death by other reasons did not influence the mOS of all patients. 
TABLE 3: Adjusted Cox regression analysis for OS and CSS of subgroups. Adjusted for age, gender, race, year of diagnosis, grade, AJCC T stage, AJCC N stage, AFP, chemotherapy, surgery, marriage, tumor size, and tumor number before PSM.

\begin{tabular}{|c|c|c|c|c|}
\hline \multirow{2}{*}{ Characteristics } & \multicolumn{2}{|c|}{ OS } & \multicolumn{2}{|c|}{ CSS } \\
\hline & HR (95\% CI) & $P$ value & HR $(95 \% \mathrm{CI})$ & $P$ value \\
\hline With bone metastases & & 0.005 & & $<0.001$ \\
\hline Radiotherapy & Reference & & Reference & \\
\hline Nonradiotherapy & $1.223(1.062,1.410)$ & & $1.326(1.138,1.545)$ & \\
\hline With lung metastases & & 0.020 & & 0.088 \\
\hline Radiotherapy & Reference & & Reference & \\
\hline Nonradiotherapy & $1.394(1.053,1.846)$ & & $1.305(0.961,1.773)$ & \\
\hline With multiorgan metastases & & 0.020 & & 0.038 \\
\hline Radiotherapy & Reference & & Reference & \\
\hline Nonradiotherapy & $1.387(1.053,1.827)$ & & $1.374(1.018,1.855)$ & \\
\hline Fibrosis scores $0-4$ & & 0.630 & & 0.165 \\
\hline Radiotherapy & Reference & & Reference & \\
\hline Nonradiotherapy & $1.153(0.647,2.053)$ & & $2.769(0.657,4.797)$ & \\
\hline Fibrosis scores 5-6 & & 0.100 & & 0.322 \\
\hline Radiotherapy & Reference & & Reference & \\
\hline Nonradiotherapy & $1.255(0.957,1.645)$ & & $1.328(0.758,2.327)$ & \\
\hline
\end{tabular}

Univariable regression analysis was not conducted in the current study due to the large sample size of the study; and, in the multivariate regression analysis, after excluding potential confounding factors, the patients who did not receive radiotherapy still had higher all-cause mortality rate and cancer-specific mortality rate, indicating that radiotherapy prolongs the survival of HCC patients with metastases to different extrahepatic organs.

Previous study has documented that patients with metastases to different organs and different liver function status had different survival times [30, 31]. Therefore, subgroup analysis was conducted in the present study to explore whether radiotherapy improved the survival of patients with metastases to different organs and with different fibrosis scores. The Kaplan-Meier analysis showed that the mOS and mCSS of patients with bone metastases and multiorgan metastases were longer in patients treated with radiotherapy than in the nonradiotherapy group. Additionally, the adjusted Cox proportional risk model showed that HCC patients with bone metastases and multiorgan metastases who did not receive radiotherapy had higher all-cause mortality and cancer-specific mortality rates than patients who received radiotherapy. The evaluation of the efficacy of radiotherapy in patients with brain metastases was not conducted here because the number of these patients was small, which might lead to unreliable conclusions. However, in the study, the fibrosis scores of patients did not influence the survival of all patients because the multivariable regression analysis showed that patients with fibrosis scores of 5-6 did not have higher all-cause mortality rate and cancerspecific mortality rate than patients with fibrosis sores of $0-4$; and, in the subgroups analysis, radiotherapy did not prolong the survival of patients compared to no radiotherapy, which might show that the liver function of patients might not influence the survival of patients in the current study. However, the fibrosis scores are not a recognized indicator of liver function. Future studies are needed to include Child-Pugh score to confirm the results of the study. The results of subgroup analysis showed that HCC patients with bone metastases and multiorgan metastases could obtain more survival benefits from radiotherapy.

Patients with advanced HCC are recommended to receive atezolizumab plus bevacizumab, sorafenib, and lenvatinib as their first-line treatments and regorafenib, cabozantinib, and ramucirumab as their secondary treatments [8, 32-34]. However, the adverse events of these treatments are high and some parts of patients cannot tolerate it. For these patients, there are no specific treatments recommended. Besides, there are few studies focusing on the systemic therapies on the treatments for HCC patients with extrahepatic metastases. Thus, at present, the results of the study might provide new evidence that HCC patients with extrahepatic metastases could get survival benefits from radiotherapy.

This study has some limitations. First, it was designed as a retrospective study, which might have led to the selection bias. However, selection bias was minimized by conducting PSM. Second, the study did not consider physical condition of patients because the SEER database does not provide this information. Future studies should include these factors to further strengthen the conclusions of the present analysis.

\section{Conclusion}

This study included a large number of HCC patients with extrahepatic metastases, treated or not treated with radiotherapy. The performed analyses documented that radiotherapy-treated HCC patients with bone metastases or multiorgan metastases had longer survival time than patients who were not subjected to radiotherapy. The study provides evidence that can be used clinically to select the best treatment for these patients.

\section{Abbreviations}

HCC: Hepatocellular carcinoma

SEER: Surveillance, epidemiology, and end results database

OS: Overall survival 
CSS: Cancer-specific survival

PSM: Propensity score matching

AJCC: American joint committee on cancer

EASL: European association for the study of the liver.

\section{Data Availability}

The data used in the study are available from SEER database (https://seer.cancer.gov/data/) (accession number: 12577Nov2019).

\section{Additional Points}

Guidelines for Methods.

\section{Ethical Approval}

The study was approved by the Ethics Committee board of Tongji Medical College, Huazhong University of Science and Technology. This study was carried out in compliance with the Helsinki Declaration. The requirement of informed consent was waived by the board of Tongji Medical College, Huazhong University of Science and Technology, because the study utilized the SEER database.

\section{Conflicts of Interest}

All the authors declare that there are no conflicts of interest.

\section{Authors' Contributions}

Chuansheng Zheng and Yiming Liu conceived and designed the study. Chuansheng Zheng provided administrative support. Lei Chen and Zhiwen Wang performed data collection. Lei Chen, Yanqiao Ren, Songlin Song, and Tao Sun performed data analysis. Zhiwen Wang, Songlin Song, Weihua Zhang, and Lei Chen wrote the manuscript. Chuansheng Zheng and Yiming Liu wrote the manuscript. All the authors approved the manuscript. The authors Lei Chen, Zhiwen Wang, and Songlin Song contributed equally to the manuscript.

\section{Acknowledgments}

This study was supported by the National Natural Science Foundation of China (nos. 81873919 and 81801810).

\section{Supplementary Materials}

Table 1: adjusted Cox regression analysis for OS and CSS of subgroups (adjusted for age, gender, race, year of diagnosis, grade, AJCC T stage, AJCC N stage, surgery, marriage, tumor size, and tumor number after PSM). Figure 1: KaplanMeier curves of OS and CSS in subgroups before PSM. (A-B) Kaplan-Meier curves of OS and CSS in patients with bone metastases; (C-D) Kaplan-Meier curves of OS and CSS in patients with lung metastases; (E-F) Kaplan-Meier curves of OS and CSS in patients with multiorgan metastases; (G-H) Kaplan-Meier curves of OS and CSS in patients with fibrosis scores of 0-4 and Kaplan-Meier curves of OS and CSS in patients with fibrosis scores of 5-6. Figure 2: Kaplan-Meier curves of OS and CSS in subgroups after PSM. (A-B) Kaplan-Meier curves of OS and CSS in patients with bone metastases; (C-D) Kaplan-Meier curves of OS and CSS in patients with lung metastases; (E-F) Kaplan-Meier curves of OS and CSS in patients with multiorgan metastases; (G-H) Kaplan-Meier curves of OS and CSS in patients with fibrosis scores of 0-4 and Kaplan-Meier curves of OS and CSS in patients with fibrosis scores of 5-6. (Supplementary Materials)

\section{References}

[1] F. Bray, J. Ferlay, I. Soerjomataram, R. L. Siegel, L. A. Torre, and A. Jemal, "Global cancer statistics 2018: GLOBOCAN estimates of incidence and mortality worldwide for 36 cancers in 185 countries," CA: A Cancer Journal for Clinicians, vol. 68, no. 6, pp. 394-424, 2018.

[2] Z. Chen, H. Xie, M. Hu et al., "Recent progress in treatment of hepatocellular carcinoma," American journal of Cancer Research, vol. 10, no. 9, pp. 2993-3036, 2020.

[3] Y. Kawaguchi, H. A. Lillemoe, and J.-N. Vauthey, "Surgical resection," Clinics in Liver Disease, vol. 24, no. 4, pp. 637-655, 2020.

[4] P. J. Thuluvath, C. To, and W. Amjad, "Role of locoregional therapies in patients with hepatocellular cancer awaiting liver transplantation," American Journal of Gastroenterology, vol. 116, no. 1, pp. 57-67, 2021.

[5] T. C. Hui, J. Kwan, and U. Pua, "Advanced techniques in the percutaneous ablation of liver tumours," Diagnostics (Basel, Switzerland), vol. 11, no. 4, 2021.

[6] J.-L. Raoul, B. Sangro, A. Forner et al., "Evolving strategies for the management of intermediate-stage hepatocellular carcinoma: available evidence and expert opinion on the use of transarterial chemoembolization," Cancer Treatment Reviews, vol. 37, no. 3, pp. 212-220, 2011.

[7] T. Yang, W. Qin, X. Sun et al., "Efficacy and safety of drugeluting bead-transcatheter arterial chemoembolization using 100-300 $\mu \mathrm{m}$ versus $300-500 \mu \mathrm{m}$ CalliSpheres microspheres in patients with advanced-stage hepatocellular carcinoma," Journal of Cancer Research and Therapeutics, vol. 16, no. 7, pp. 1582-1587, 2020.

[8] EASL Clinical Practice Guidelines, "Management of hepatocellular carcinoma," Journal of Hepatology, vol. 69, no. 1, pp. 182-236, 2018.

[9] J. M. Llovet, S. Ricci, V. Mazzaferro et al., "Sorafenib in advanced hepatocellular carcinoma," New England Journal of Medicine, vol. 359, no. 4, pp. 378-390, 2008.

[10] M. Kudo, R. S. Finn, S. Qin et al., "Lenvatinib versus sorafenib in first-line treatment of patients with unresectable hepatocellular carcinoma: a randomised phase 3 non-inferiority trial," The Lancet, vol. 391, no. 10126, pp. 1163-1173, 2018.

[11] L. Bucci, F. Garuti, B. Lenzi et al., "The evolutionary scenario of hepatocellular carcinoma in Italy: an update," Liver International, vol. 37, no. 2, pp. 259-270, 2017.

[12] S. Loibl, P. Poortmans, M. Morrow, C. Denkert, and G. Curigliano, "Breast cancer," Lancet (London, England), vol. 397, no. 10286, pp. 1750-1769, 2021.

[13] E. J. Buss, L. A. Kachnic, and D. P. Horowitz, "Radiotherapy for locally advanced pancreatic ductal adenocarcinoma," Seminars in Oncology, vol. 48, no. 1, 2021. 
[14] P. Rogowski, M. Roach, N.-S. Schmidt-Hegemann et al., "Radiotherapy of oligometastatic prostate cancer: a systematic review," Radiation Oncology, vol. 16, no. 1, p. 50, 2021.

[15] A. Hijab, B. Tocco, I. Hanson et al., "MR-guided adaptive radiotherapy for bladder cancer," Frontiers in Oncology, vol. 11, Article ID 637591, 2021.

[16] J. Lin, H. Jiang, W. Yang et al., "Predictive factors of benefit from iodine-125 brachytherapy for hepatocellular carcinoma with portal vein tumor thrombosis," Brachytherapy, vol. 18, no. 2, pp. 233-239, 2019.

[17] D. Yuan, Z. Gao, J. Zhao, H. Zhang, and J. Wang, "125I seed implantation for hepatocellular carcinoma with portal vein tumor thrombus: a systematic review and meta-analysis," Brachytherapy, vol. 18, no. 4, pp. 521-529, 2019.

[18] K. Ito, S. Sugita, Y. Nakajima et al., "Electron beam intraoperative radiotherapy for metastatic epidural spinal cord compression: a prospective observational study," Clinical \& Experimental Metastasis, vol. 38, no. 2, pp. 219-225, 2021.

[19] Y. Mukai, N. R. Yokota, M. Sugiura et al., "Outcome of radiation therapy for stage IVB uterine cervical cancer with distant lymph nodes metastases; sequential irradiation for distant lymph nodes metastases," In Vivo, vol. 35, no. 2, pp. 1169-1176, 2021.

[20] P. B. Romesser, B. P. Neal, and C. H. Crane, "External beam radiation therapy for liver metastases," Surgical Oncology Clinics of North America, vol. 30, no. 1, pp. 159-173, 2021.

[21] D. A. Palma, R. Olson, S. Harrow et al., "Stereotactic ablative radiotherapy versus standard of care palliative treatment in patients with oligometastatic cancers (SABR-COMET): a randomised, phase 2, open-label trial," The Lancet, vol. 393, no. 10185, pp. 2051-2058, 2019.

[22] X. Wei, Y. Jiang, X. Zhang et al., "Neoadjuvant three-dimensional conformal radiotherapy for resectable hepatocellular carcinoma with portal vein tumor thrombus: a randomized, open-label, multicenter controlled study," Journal of Clinical Oncology, vol. 37, no. 24, pp. 2141-2151, 2019.

[23] P. K. H. Chow, M. Gandhi, S.-B. Tan et al., "SIRveNIB: selective internal radiation therapy versus sorafenib in asiapacific patients with hepatocellular carcinoma," Journal of Clinical Oncology, vol. 36, no. 19, pp. 1913-1921, 2018.

[24] J. Ricke, H. J. Klümpen, H. Amthauer et al., "Impact of combined selective internal radiation therapy and sorafenib on survival in advanced hepatocellular carcinoma," Journal of Hepatology, vol. 71, no. 6, pp. 1164-1174, 2019.

[25] D. A. Palma, R. Olson, S. Harrow et al., "Stereotactic ablative radiotherapy for the comprehensive treatment of oligometastatic cancers: long-term results of the SABR-COMET phase II randomized trial," Journal of Clinical Oncology, vol. 38, no. 25, pp. 2830-2838, 2020.

[26] R. Phillips, W. Y. Shi, M. Deek et al., "Outcomes of observation vs stereotactic ablative radiation for oligometastatic prostate cancer: the ORIOLE phase 2 randomized clinical trial," JAMA Oncology, vol. 6, no. 5, pp. 650-659, 2020.

[27] J. Sun, L. Yang, J. Shi et al., "Postoperative adjuvant IMRT for patients with HCC and portal vein tumor thrombus: an openlabel randomized controlled trial," Radiotherapy and Oncology, vol. 140, pp. 20-25, 2019.

[28] K. Schütte, R. Schinner, M. P. Fabritius et al., "Impact of extrahepatic metastases on overall survival in patients with advanced liver dominant hepatocellular carcinoma: a subanalysis of the SORAMIC trial," Liver Cancer, vol. 9, no. 6, pp. 771-786, 2020.
[29] T. H. Kim, S. Park, C. H. Rim, C. Choi, and J. Seong, "Improved oncologic outcomes by ablative radiotherapy in patients with bone metastasis from hepatocellular carcinoma," Journal of Cancer Research and Clinical Oncology, vol. 147, no. 9, 2021.

[30] I. Poon, D. Erler, R. Dagan et al., "Evaluation of definitive stereotactic body radiotherapy and outcomes in adults with extracranial oligometastasis," JAMA Network Open, vol. 3, no. 11, Article ID e2026312, 2020.

[31] A. Granito and L. Bolondi, "Non-transplant therapies for patients with hepatocellular carcinoma and Child-PughTurcotte class B cirrhosis," The Lancet Oncology, vol. 18, no. 2, pp. e101-e112, 2017.

[32] L. Ielasi, V. Sansone, A. Granito, F. Benevento, S. De Lorenzo, and F. Tovoli, "An update of treatments of hepatocellular carcinoma in patients refractory to sorafenib," Drugs of Today (Barcelona, Spain: 1998), vol. 54, no. 10, pp. 615-627, 2018.

[33] A. Granito, A. Forgione, S. Marinelli et al., "Experience with regorafenib in the treatment of hepatocellular carcinoma," Therapeutic Advances in Gastroenterology, vol. 14, Article ID $17562848211016959,2021$.

[34] J. M. Llovet, R. K. Kelley, A. Villanueva et al., "Hepatocellular carcinoma," Nature Reviews Disease primers, vol. 7, no. 1, p. 6, 2021. 